\title{
Characterizing Sulfur in TEM and STEM, with Applications to Lithium Sulfur Batteries
}

\author{
Barnaby D.A. Levin ${ }^{1}$, Michael J. Zachman ${ }^{1}$, Jörg G. Werner ${ }^{2}$, Ulrich Wiesner ${ }^{2}$, Lena F. Kourkoutis ${ }^{1,3}$, \\ David A. Muller ${ }^{1,3}$ \\ 1. Department of Applied and Engineering Physics, Cornell University, Ithaca, NY, USA, 14853 \\ 2. Department of Materials Science, Cornell University, Ithaca, NY, USA, 14853 \\ ${ }^{3 .}$ Kavli Institute for Nanoscale Science, Cornell University, Ithaca, NY, USA, 14853
}

The lithium sulfur (Li-S) battery is a promising technology with the potential to provide greater energy density at lower cost than current lithium ion batteries. One of the main challenges to improving the performance of Li-S batteries is the dissolution and loss of sulfur to the electrolyte as the battery is cycled [1]. Recently much effort has focused on nanostructured electrodes that could sequester the sulfur and prevent its loss during battery operation. Analyzing the distribution of sulfur in these electrodes is critical for creating durable Li-S batteries with high energy density.

Reliable characterization of sulfur electrodes by TEM is presently problematic due to Sulfur sublimation. With a vapor pressure of $\sim 6 \times 10^{-7}$ Torr at $18^{\circ} \mathrm{C}$ [2], Sulfur typically sublimates in the sub- $10^{-7}$ Torr vacuum of a TEM. A time series of a sulfur particle sublimating in TEM is shown in Fig. 1. The observed sublimation rate of $\sim 10^{15} \mathrm{~S}$ atoms $\mathrm{cm}^{-2} \mathrm{~s}^{-1}$ ( $\sim$ one monolayer of $\mathrm{S}$ per second) is consistent with bulk measurements [3]. Sulfur encapsulated inside spheres of other materials, or in micropores, can remain stable enough in vacuum to image [4, 5]. However, the sublimation of un-encapsulated sulfur is a serious problem for characterizing Li-S battery materials because one cannot be certain that all of the sulfur that was originally in a sample is still present when imaging. For example, in Fig. 2 a) spheres of polyacrylonitrile (PAN), intended for a sulfur electrode, are imaged at $18^{\circ} \mathrm{C}$. Sulfur is detected inside one sphere, but is absent from the others. This may be due to some aspect of the sample synthesis, or sublimation of sulfur from the pores visible in the empty PAN shells. Any S outside of the spheres would have sublimated away prior to imaging. The $\mathrm{S}$ distribution in this sample (prior to exposure to vacuum) cannot be inferred from this image.

Cryo-TEM can suppress detrimental sulfur sublimation by cooling the sample to temperatures far below that of the sulfur sublimation line (Fig. 2 c)). A sulfur particle imaged at $\sim-175^{\circ} \mathrm{C}$ using a Gatan 626 cryo-holder is shown in Fig. 2 d). After over 4 hours in vacuum, there was no detectable sulfur mass loss. Indeed, the sulfur is stable enough under the beam to allow for EDX mapping (Fig. $2 \mathrm{f}$ )). This demonstrates that cryo-TEM can allow imaging of the inherent sulfur distribution in materials such as Li-S battery electrodes.

\section{References:}

[1] P. G. Bruce, et. al. Nature Materials, 11, 1, (2011) p. 19-29.

[2] A.G.M. Ferreira and L.Q. Lobo, J. Chem. Thermodynamics 43 (2011), p. 95-104.

[3] D.B. Nash, Icarus, 72 (1987), p. 1-34. 
[4] Z. Wei Seh, et.al. Nature Communications, 4, (2013) p. 1331. [5] D.W. Wang et. al. Physical Chemistry Chemical Physics, 14, 24, (2012) p. 8703. [6] Work supported by the Energy Materials Center at Cornell, DOE EFRC BES (DE-SC0001086). EM Facility support from NSF MRSEC program (DMR 1120296).
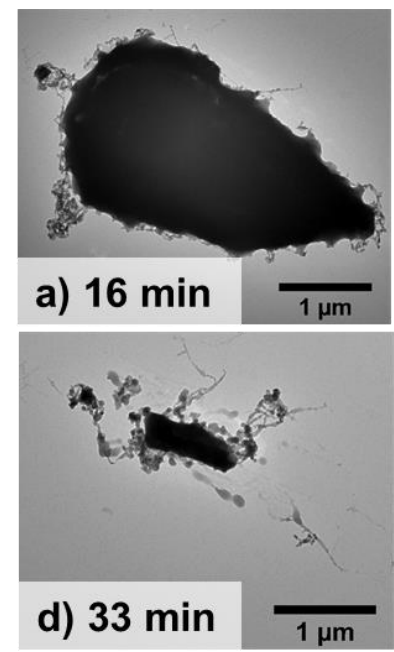
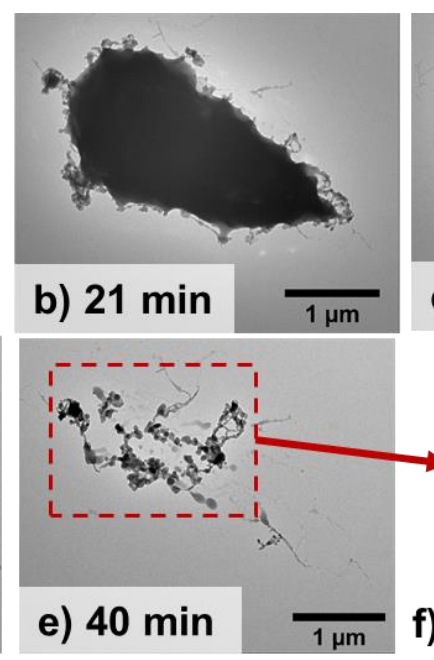
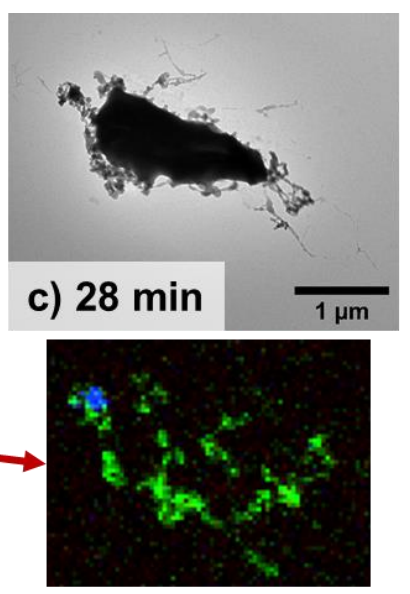

f) Red: Carbon Green: Sulfur

Figure 1. a)-e) Time series of sulfur particle sublimating in FEI Tecnai F-20 TEM. Majority of sulfur particle has sublimated by 40 minutes. Ambient temperature $18^{\circ} \mathrm{C}$. Ambient pressure $8.8 \times 10^{-8}$ Torr. Beam dose, $10 \mathrm{e}^{-} \mathrm{nm}^{-2} \mathrm{~s}^{-1}$. Exposure time per image, 2s. Sample not exposed to beam between images. f) STEM EDX of sulfur residue after $\sim 3$ hours. Appearance of residue unchanged from 40 minutes. Residue identified as low vapor pressure sulfur polymer [3].

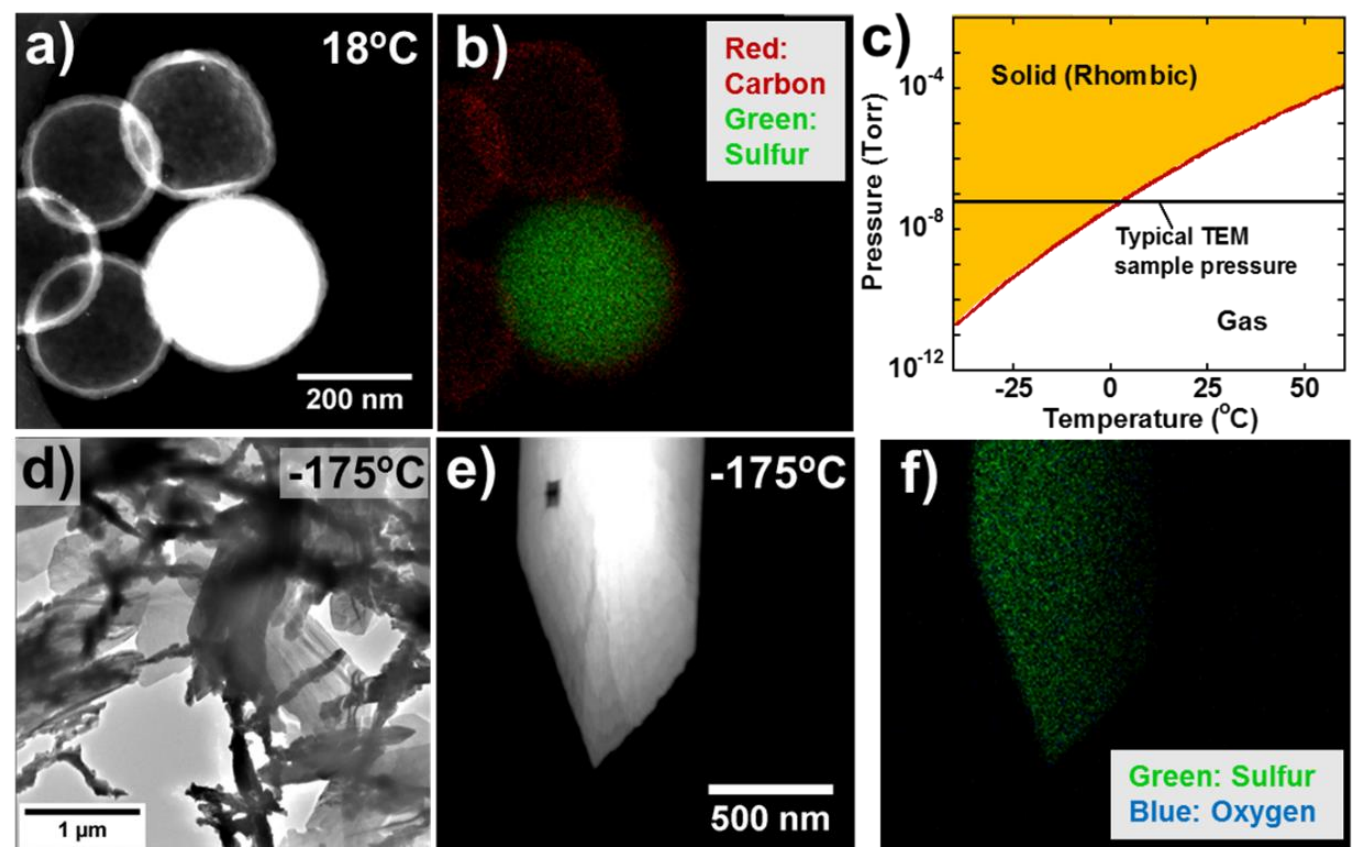

Figure 2. a) HAADF image of sulfur encapsulated in PAN, at room temperature. b) Corresponding EDX map. Spheres which appear to have pores contain no sulfur. Sample synthesis by Weidong Zhou, Cornell. c) Sulfur vapor pressure curve [2]. d) Sulfur in cryo-TEM. Branch-like structures were not visible at room temperature. e) HAADF image of tip of a sulfur particle, after 4 hrs in cryo-TEM. f) Corresponding EDX map. Trace of oxygen from minor ice contamination. 